\title{
Passive Control of Nanoparticles on MHD Jeffrey Nanofluid past a Convectively Heated Moving Plate with Thermal Radiation
}

\author{
S. Mohd Zokri, N. S. Arifin, A. R. Mohd Kasim and M. Z. Salleh* \\ Faculty of Industrial Science \& Technology, Universiti Malaysia Pahang, \\ 26300 UMP Kuantan, Pahang, Malaysia \\ *Email: zuki@ump.edu.my \\ Phone: +6095492203; Fax: +6095492766
}

\begin{abstract}
A theoretical study is conducted to investigate the thermal radiation effect on boundary layer flow of magneto-hydrodynamic (MHD) Jeffrey nanofluid across a moving plate with convective boundary condition. More physically acceptable model of passively controlled wall nanoparticle concentration is executed. Similarity transformation variables are utilised to transform the partial differential equations to non-linear ordinary differential equations. An effective Runge-Kutta Fehlberg Fourth-Fifth order (RKF45) method is employed to solve the obtained equations numerically. Validation of the present results has been made with the existing studies under the limiting cases and the results are found to be in a good agreement. Numerical solutions for several pertinent parameters are provided graphically over specified distributions. The results indicate that the temperature profile intensifies attributable to the increasing thermal radiation parameter. Besides, the increase of Brownian motion parameter pronounces negligible effect on the temperature profile, whereas nanoparticle concentration profile declines. Moreover, increment in the thermophoresis diffusion parameter results in the escalation of the temperature and nanoparticle concentration profiles.
\end{abstract}

Keywords: Passive control of nanoparticles; Jeffrey nanofluid; MHD; moving plate; thermal radiation; convective boundary condition.

\section{INTRODUCTION}

Fluids which are no longer adequate to be described by the well-known Navier-Stokes equations in an appropriate way are termed as non-Newtonian fluids. In contrast to viscous fluids, the rheological properties of every non-Newtonian fluids are dissimilar. In fact, they are characterised by different models such as Williamson fluid [1], viscoelastic fluid [2], Jeffrey fluid [3], nanofluid [4] and micropolar fluid [5]. Among the suggested non-Newtonian fluid models, the model of Jeffrey fluid is preferable for its ability of describing dual impacts of relaxation and retardation times. This fluid model is categorised as a simple and linear model that exploits time derivatives as opposed to convected derivatives.

Nevertheless, there exists a high apprehension over the unsatisfactory thermal conductivity of Jeffery fluid. As with the modern development of engineering technology, the idea of suspended nanoparticles (nanofluid) has been publicised as a means for reinforcing the optical properties of conventional fluids. In nanofluid, Brownian motion of nanoparticles is the fundamental aspect that assists in the improvement of thermal conductivity. Such improvement is vastly important in heat transfer escalation especially 
in industrial and metallurgical applications such as air-conditioning, transportation, power generation, ventilation, heating and cooling. Dalir et al. [6] considered the Jeffrey nanofluid model past a stretching sheet with entropy generation and viscous dissipation effects. Later, the fluid flow over a radial sheet in Jeffrey nanofluid was studied by Ashraf et al. [7] in the presence of thermal radiation effect. Narayana and Babu [8] addressed the effects of thermal radiation and chemical reaction over a stretching sheet in the presence of power law form. The solution was then attained numerically via the Runge-Kutta fourth order scheme. Very recently, Khan et al. [9] and Zokri et al. [10] investigated the Jeffrey nanofluid induced by inclined stretching sheet and moving plate, respectively.

Nield and Kuznetsov [11] explained that the nanoparticle fraction at the boundary can be controlled in equivalent way as the temperature. However, in practice, there is no evidence in what manner it could possibly be done. The problem was then reviewed with a more physically realistic and acceptable model that accounts both nanofluids parameters, i.e. the Brownian motion and the thermophoresis diffusion parameters [12]. Here, the condition of zero normal flux at the moving plate was interpreted to dispel nanoparticle away from the plate and that the value there alters appropriately. Zaidi and Mohyud-Din [13] incorporated the passive control condition of nanoparticles at the boundary to scrutinise the laminar wall jet flow of nanofluid past a narrow vertical slit. Ibrahim and Haq [14] considered the zero normal flux condition at the wall of the stretched sheet with convective heating and magneto-hydrodynamic (MHD) effects. Similar condition was also integrated by Jahan et al. [15] in a nanofluid from a stretching/shrinking sheet. Interestingly, they performed stability analysis to determine the physically stable and practicable numerical solutions. Halim et al. [16] analysed both active and passive control conditions of nanoparticles from a stagnation point flow of slipped stretched surface in a Maxwell fluid. More recently, Jahan et al. [17] extended their previous work (Jahan et al. [15]) to conduct the stability analysis of a nanofluid flow past a moving surface.

Flow past a moving plate is in the limelight owing to its vital occurrence in industrial and engineering disciplines, principally in metal and polymer extrusion, calculation of drag for plate at zero incidence, heat-treated materials travelling between a feed roll and a wind-up roll, and cooling of metallic sheets or electronic chips. Sakiadis [18] reported a classical flow problem on a constant speed moving plate. Following the Sakiadi's theoretical predictions, Tsou et al. [19] conducted a combined analytical and experimental study in both laminar and turbulent flow conditions. Ever since, such problem has been broadened to various fluids, boundary conditions and effects [20-23]. In particular, Mohamed et al. [24] examined the flow problem in a nanofluid with frictional heating while Satish and Venkatasubbaiah [25] deliberated the turbulent forced convection in a channel flow. In these two studies, the numerical approach was adopted where the respective Keller-box method and Runge Kutta Fehlberg method were executed. Soon after, Soomro et al. [26] studied the nonlinear thermal radiation past a moving surface in a Sisko fluid. The exploration of the thermal management was achieved through melting heat transfer, followed by implementation of the Collocation method on the resulting ordinary differential equations. Another endeavour in this area was documented by Nandeppanavar [27] to investigate the Casson fluid flow past a melting sheet moving parallel to a melting stream.

The aforementioned literatures have evidently revealed that the Jeffrey nanofluid model was thoroughly explored from a stretching sheet, while mainly there was one study conducted from a moving plate. The passive control of nanoparticles, on the other hand was identified to greatly deliberate on the Newtonian fluid (nanofluid) rather than the 
non-Newtonian fluid model suspended with nanoparticles. Motivated by the realistic approach of passively controlled nanoparticles, the present study caters the problem of MHD Jeffrey nanofluid passing through a convectively heated moving plate with thermal radiation and passive control condition. The knowledge of the radiative heat transfer in the system is vital as the quality of the final product is prominently influenced by the heat controlling factors. Several recent contributions dealing with the thermal radiation effect embrace those of Afridi and Qasim [28], and Narayana and Babu [29].

\section{MATHEMATICAL FORMULATION}

\section{Basic Principle of Fluid Dynamics}

The basic principle of computational fluid dynamics lies on the succeeding fundamental governing equations:

\section{Continuity equation}

The principle of conservation of mass states that mass is conserved; to be precise, the difference between the inflow and outflow of matters must be equivalent to the increase in fluid mass contained in the control volume. This statement necessitates an establishment of the continuity equation to elucidate the relationship between the fluid density and fluid velocity at any point [30].

$$
\frac{\partial \rho_{f}}{\partial t}+\nabla \cdot \rho_{f} \mathbf{V}=0
$$

where $\rho_{f}$ is fluid density, $t$ is time, $\nabla=\left(\frac{\partial}{\partial x}, \frac{\partial}{\partial y}, \frac{\partial}{\partial z}\right)$ is operator and $\mathbf{V}=(u, v, w)$ is velocity vector of the flow field. The fluid density is constant for incompressible fluid, then Eq. (1) becomes:

$$
\nabla \cdot \mathbf{V}=0
$$

\section{Momentum equation}

The law of conservation of momentum is derived from the Newton's second law of motion which simply states that the resulting force acting on the control volume is equal to the rate of change of momentum. This can be symbolised as

$$
\mathbf{F}=m \mathbf{a}
$$

where $\mathbf{F}$ implies force exerted on the fluid element, $m$ denotes mass of the element and $\mathbf{a}$ is acceleration of the element. Then, the momentum equation for the Jeffrey fluid can be expressed in differential form as $[30,31]$ :

$$
\rho_{f} \frac{D \mathbf{V}}{D t}=\nabla \cdot \mathbf{T}+\mathbf{F}_{b}
$$




$$
\begin{aligned}
& \mathbf{T}=-p \mathbf{I}+\mathbf{S} \\
& \mathbf{I}=\left(\begin{array}{lll}
1 & 0 & 0 \\
0 & 1 & 0 \\
0 & 0 & 1
\end{array}\right) \\
& \mathbf{S}=\frac{\mu}{1+\lambda}\left[\mathbf{R}_{1}+\lambda_{1}\left(\frac{\partial \mathbf{R}_{1}}{\partial t}+\mathbf{V} \cdot \nabla\right) \mathbf{R}_{1}\right] \\
& \mathbf{R}_{1}=(\nabla \mathbf{V})+(\nabla \mathbf{V})^{t}
\end{aligned}
$$

where $\frac{D}{D t}=\frac{\partial}{\partial t}+u \frac{\partial}{\partial x}+v \frac{\partial}{\partial y}+w \frac{\partial}{\partial z}$ denotes material derivative, $\mathbf{T}$ implies Cauchy stress tensor for Jeffrey fluid, $p$ is scalar pressure, $\mathbf{I}$ is identity tensor, $\mathbf{S}$ is extra stress tensor, $\mathbf{R}_{1}$ is Rivlin-Ericksen tensor, $\mu$ is dynamic viscosity and $\lambda$ and $\lambda_{1}$ are material parameters of Jeffrey fluid called as the ratio of relaxation to retardation times and retardation time, respectively. Furthermore, the body force $\mathbf{F}_{b}=\left(F_{b x}, F_{b y}, F_{b z}\right)$ is expressed as:

$$
\mathbf{F}_{b}=\mathbf{g}+\mathbf{J} \times \mathbf{B}
$$

where $\mathbf{J}=\sigma(\mathbf{E}+\mathbf{V} \times \mathbf{B})$ is current density, $\mathbf{g}$ is gravitational acceleration and $\mathbf{B}=\mathbf{B}_{\mathbf{0}}(x)+\mathbf{b}$ is magnetic force where $\mathbf{B}_{\mathbf{0}}(x)=\left(0, B_{0}(x), 0\right)$ implies the magnetic field and $\mathbf{b}$ denotes induced magnetic field. In the present study, $\mathbf{g}$ and $\mathbf{b}$ are neglected under respective consideration of forced convection flow and assumption of small magnetic Reynolds number value [32]. In addition, $\mathbf{E}$ disappears everywhere due to the presence of electrically insulating boundary around the plate [33].

\section{Energy equation}

The derivation of the energy equation originates from the principle of conservation of energy (first law of thermodynamics) which states that energy cannot be created nor destroyed. As in Buongiorno [34], the energy equation can be expressed as

$$
(\rho c)_{f} \frac{D T}{D t}=-\nabla \cdot \mathbf{q}+h_{p} \nabla \cdot \mathbf{j}_{p}-\nabla \cdot \mathbf{q}_{r}
$$

where $(\rho c)_{f}$ is heat capacity of the base fluid, $T$ is fluid temperature and $h_{p}$ is specific enthalpy of the nanoparticle material. Further, $\mathbf{q}$ is energy flux relative to the frame moving with the nanofluid velocity, $\mathbf{q}_{r}$ is Rosseland approximation and $\mathbf{j}_{p}$ is diffusion mass flux for the nanoparticle, given by:

$$
\mathbf{q}=-k_{f} \nabla T+h_{p} \mathbf{j}_{p}
$$


$\mathbf{q}_{r}=\frac{-4 \sigma^{*}}{3 k^{*}} \nabla T^{4}$

$\mathbf{j}_{p}=-\rho_{p} D_{B} \nabla C-\rho_{p} D_{T} \frac{\nabla T}{T_{\infty}}$

\section{Concentration equation}

The conservation of mass equation for the nanoparticle can be expressed as follows [34]:

$$
\frac{D C}{D t}=-\frac{1}{\rho_{p}} \nabla \cdot \mathbf{j}_{p}
$$

where $\mathbf{j}_{p}$ is defined by Eq. (13).

\section{Governing Equations}

A steady, two-dimensional forced convective and laminar flow of Jeffrey nanofluid past a flat plate moving with constant velocity, $u_{w}(x)$ in an electrically conducting fluid, where the magnetic field, $B_{0}$ is directed perpendicularly to the plate is deliberated. The effects of thermal radiation along with the passive control of nanoparticles are also discussed. Furthermore, $T, T_{f}$ and $T_{\infty}$ represent the respective boundary layer temperature, hot fluid temperature and ambient temperature, respectively, whereas $C$ and $C_{\infty}$ imply the nanoparticle concentration and ambient nanoparticle concentration, respectively. The flow diagram of this study is displayed in Figure 1.

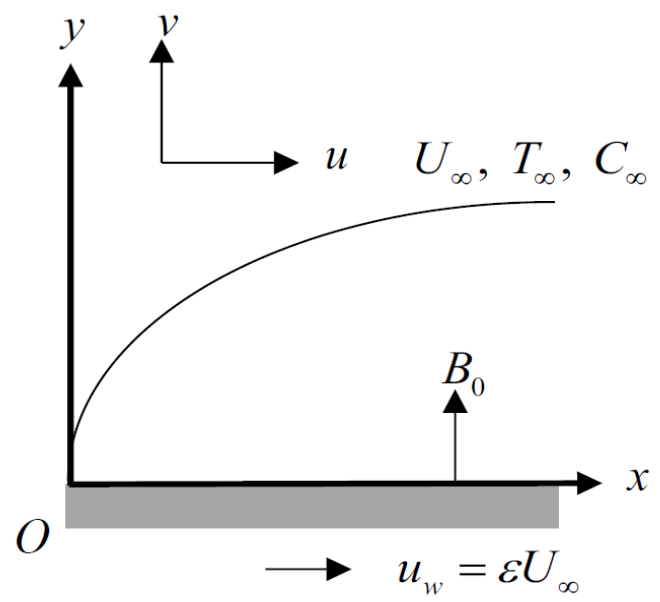

Figure 1. The flow diagram.

Assuming that the Boussinesq approximation has taken place and that the nanoparticle concentration is assumed to dilute, then a steady two-dimensional Eqs. (2), (4), (10) and (14) can be expressed as follows [3, 24]: 


$$
\begin{aligned}
& \frac{\partial u}{\partial x}+\frac{\partial v}{\partial y}=0 \\
& u \frac{\partial u}{\partial x}+v \frac{\partial u}{\partial y}=\frac{v}{1+\lambda}\left[\frac{\partial^{2} u}{\partial y^{2}}+\lambda_{1}\left(u \frac{\partial^{3} u}{\partial x \partial y^{2}}-\frac{\partial u}{\partial x} \frac{\partial^{2} u}{\partial y^{2}}+\frac{\partial u}{\partial y} \frac{\partial^{2} u}{\partial x \partial y}+v \frac{\partial^{3} u}{\partial y^{3}}\right)\right]-\frac{\sigma B_{0}^{2}}{\rho_{f}} u \\
& u \frac{\partial T}{\partial x}+v \frac{\partial T}{\partial y}=\alpha \frac{\partial^{2} T}{\partial y^{2}}-\frac{1}{(\rho c)_{f}} \frac{\partial q_{r}}{\partial y}+\tau\left[D_{B} \frac{\partial C}{\partial y} \frac{\partial T}{\partial y}+\frac{D_{T}}{T_{\infty}}\left(\frac{\partial T}{\partial y}\right)^{2}\right] \\
& u \frac{\partial C}{\partial x}+v \frac{\partial C}{\partial y}=D_{B} \frac{\partial^{2} C}{\partial y^{2}}+\frac{D_{T}}{T_{\infty}} \frac{\partial^{2} T}{\partial y^{2}}
\end{aligned}
$$

with associated boundary conditions:

$$
\begin{aligned}
& u=u_{w}(x)=\varepsilon U_{\infty}, \quad v=V_{w}, \quad-k_{f} \frac{\partial T}{\partial y}=h_{f}\left(T_{f}-T\right), D_{B} \frac{\partial C}{\partial y}+\frac{D_{B}}{T_{\infty}} \frac{\partial T}{\partial y}=0 \text { at } y=0 \\
& u \rightarrow U_{\infty}, \quad v \rightarrow 0, \quad T \rightarrow T_{\infty}, C \rightarrow C_{\infty} \text { as } y \rightarrow \infty
\end{aligned}
$$

where $u$ and $v$ are respective components of velocity in $x$ - and $y$-directions, $\varepsilon$ is plate velocity parameter, $v$ is kinematic viscosity, $V_{w}(x)$ is velocity of the suction/injection, $U_{\infty}$ is free stream velocity, $\rho_{f}$ is density of the base fluid, $\rho_{p}$ is particle density, $q_{r}$ is radiative heat flux, $h_{f}$ is coefficient of heat transfer, $\sigma$ is electrical conductivity, $\alpha=k_{f} /(\rho c)_{f}$ is thermal diffusivity, $\tau=(\rho c)_{p} /(\rho c)_{f}$ is ratio of heat capacity, where $(\rho c)_{p}$ is heat capacity of the nanoparticle, $(\rho c)_{f}$ is heat capacity of the fluid, $k_{f}$ is thermal conductivity, $D_{B}$ is Brownian diffusion coefficient and $D_{T}$ is thermophoretic diffusion coefficient. The boundary condition of $D_{B} \frac{\partial C}{\partial y}+\frac{D_{B}}{T_{\infty}} \frac{\partial T}{\partial y}=0$ embodies the condition of zero nanoparticle flux [12]. Now, the Rosseland approximation is imposed: $q_{r}=-\frac{4 \sigma^{*}}{3 k^{*}} \frac{\partial T^{4}}{\partial y}$, where $\sigma^{*}$ and $k^{*}$ are the respective Stefan-Boltzmann constant and the mean absorption coefficient, respectively. Using the Rosseland approximation [20], Eq. (17) becomes:

$$
u \frac{\partial T}{\partial x}+v \frac{\partial T}{\partial y}=\left(\frac{k}{(\rho c)_{f}}+\frac{16 \sigma^{*} T_{\infty}^{3}}{3 k^{*}(\rho c)_{f}}\right) \frac{\partial^{2} T}{\partial y^{2}}+\tau\left[D_{B} \frac{\partial C}{\partial y} \frac{\partial T}{\partial y}+\frac{D_{T}}{T_{\infty}}\left(\frac{\partial T}{\partial y}\right)^{2}\right]
$$

Let $N r=\frac{4 \sigma^{*} T_{\infty}^{3}}{k^{*} k}$ be the radiation parameter. Then, Eq. (20) results in: 
$u \frac{\partial T}{\partial x}+v \frac{\partial T}{\partial y}=\frac{k}{(\rho c)_{f}}\left(1+\frac{4 N r}{3}\right) \frac{\partial^{2} T}{\partial y^{2}}+\tau\left[D_{B} \frac{\partial C}{\partial y} \frac{\partial T}{\partial y}+\frac{D_{T}}{T_{\infty}}\left(\frac{\partial T}{\partial y}\right)^{2}\right]$

Introducing the set of similarity transformation variables [21],

$\eta=\left(\frac{U_{\infty}}{2 x v}\right)^{1 / 2} y, \quad \psi=\left(2 U_{\infty} x v\right)^{1 / 2} f(\eta), \quad \theta(\eta)=\frac{T-T_{\infty}}{T_{f}-T_{\infty}}, \quad \phi(\eta)=\frac{C-C_{\infty}}{C_{\infty}}$

From Eq. (22), $\eta, \theta$ and $\phi$ are similarity variables, dimensionless fluid temperature and nanoparticle concentration, respectively. One can discover that Eq. (15) is trivially satisfied when stream function, $\psi$ defined by $u=\partial \psi / \partial y$ and $v=-\partial \psi / \partial x$ are applied. Taking into account Eq. (22), Eqs. (16), (17) and (21) with boundary conditions (19) yield the following equation:

$$
\begin{aligned}
& f^{\prime \prime \prime}-\frac{\lambda_{2}}{2} f f^{(i v)}+(1+\lambda)\left(f f^{\prime \prime}-2 M f^{\prime}\right)=0 \\
& \frac{1}{\operatorname{Pr}}\left(1+\frac{4 N r}{3}\right) \theta^{\prime \prime}+f \theta^{\prime}+N b \theta^{\prime} \phi^{\prime}+N t \theta^{\prime 2}=0 \\
& \phi^{\prime \prime}+L e \operatorname{Pr} f \phi^{\prime}+\frac{N t}{N b} \theta^{\prime \prime}=0 \\
& f(0)=f_{w}, \quad f^{\prime}(0)=\varepsilon, \theta^{\prime}(0)=-B i(1-\theta(0)), \quad N b \phi^{\prime}(0)+N t \theta^{\prime}(0)=0 \\
& f^{\prime}(\infty) \rightarrow 1, \quad f^{\prime \prime}(\infty) \rightarrow 0, \quad \theta(\infty) \rightarrow 0, \quad \phi(\infty) \rightarrow 0
\end{aligned}
$$

Equations (23) to (26) comprises of eight parameters $\lambda_{2}, M, N b, \operatorname{Pr}, L e, N t, B i$ and $f_{w}$ named Deborah number, magnetic parameter, Brownian motion parameter, Prandtl number, Lewis number, thermophoresis parameter, Biot number and mass suction/injection, respectively, that can be denoted as:

$$
\begin{aligned}
& \lambda_{2}=\frac{\lambda_{1} U_{\infty}}{x}, M=\frac{\sigma x B_{0}^{2}}{U_{\infty} \rho_{f}}, N b=\frac{\tau D_{B} C_{\infty}}{v}, \operatorname{Pr}=\frac{v}{\alpha}, L e=\frac{\alpha}{D_{B}}, N t=\frac{\tau D_{T}\left(T_{w}-T_{\infty}\right)}{v T_{\infty}}, \\
& B i=\frac{h_{f}}{k_{f}}\left(\frac{v}{a}\right)^{1 / 2}, f_{w}=-V_{w}\left(\frac{2}{a v}\right)^{1 / 2}
\end{aligned}
$$

where $f_{w}>0$ is mass suction while $f_{w}<0$ is mass injection. The local skin friction coefficient $C_{f}$ and the local Nusselt number $N u_{x}$ can be written as:

$$
C_{f}=\frac{\tau_{w}}{\rho u_{e}^{2}}, N u_{x}=\frac{x q_{w}}{k\left(T_{w}-T_{\infty}\right)}, S h_{x}=\frac{x j_{w}}{D_{B}\left(C_{w}-C_{\infty}\right)}
$$


where the surface shear stress $\tau_{w}$, the surface heat flux $q_{w}$ and the surface mass flux $j_{w}$ are defined as:

$$
\begin{aligned}
& \tau_{w}=\left(\frac{\mu}{(1+\lambda)}\right)\left[\left(\frac{\partial u}{\partial y}\right)+\lambda_{1}\left(u\left(\frac{\partial^{2} u}{\partial x \partial y}\right)+v\left(\frac{\partial^{2} u}{\partial y^{2}}\right)\right)\right]_{y=0}, \\
& q_{w}=-k\left(\frac{\partial T}{\partial y}\right)_{y=0}, j_{w}=-D_{B}\left(\frac{\partial C}{\partial y}\right)_{y=0}
\end{aligned}
$$

with $\mu=\rho v$ signifying the dynamic viscosity. Imposing Eq. (22) into Eq. (28) yields:

$$
\begin{aligned}
& C_{f r}=C_{f}\left(2 \operatorname{Re}_{x}\right)^{1 / 2}=\frac{1}{(1+\lambda)}\left[f^{\prime \prime}(0)-\frac{\lambda_{2}}{2} f f^{\prime \prime \prime}\right], \\
& N u r=N u_{x}\left(\frac{\operatorname{Re}_{x}}{2}\right)^{-1 / 2}=-\theta^{\prime}(0), S h r=S h_{x}\left(\frac{\operatorname{Re}_{x}}{2}\right)^{-1 / 2}=-\phi^{\prime}(0)=0,
\end{aligned}
$$

provided that the Reynold number, $\operatorname{Re}_{x}=\frac{U_{\infty} x}{v}$. It is worth mentioning that the Sherwood number, $S h r$ becomes zero resulting from the passive control condition of nanoparticles [12].

\section{NUMERICAL PROCEDURE}

\section{Runge-Kutta Fehlberg Fourth-Fifth Order (RKF45) Method}

The numerical solutions of boundary value problem of Eqs. (23)-(26) are obtained via a well-tested method for its accuracy and robustness, namely the RKF45 method. This method is basically programmed in the Maple software using a built-in function, dsolve command. The idea of this method lies on the appropriate selection of the step size, $h$ during the computation in order to acquire a good estimation of the expected error, $R$. At every step, the computation and comparison between two dissimilar approximations are conducted. If $R$ is larger than the error tolerance $\varepsilon$, the step size has to be decreased and the present step needs to be recalculated. Alternatively, if $R$ is lesser than $\varepsilon$, the present step has to be maintained and the step size needs to be enlarged for the subsequent step. The formula for the RKF45 method along with its numerical algorithm can be delineated as the following $[35,36]$ :

$$
\begin{aligned}
& k_{1}=h f\left(x_{i}, y_{i}\right), \\
& k_{2}=h f\left(x_{i}+\frac{1}{4} h, y_{i}+\frac{1}{4} k_{1}\right), \\
& k_{3}=h f\left(x_{i}+\frac{3}{8} h, y_{i}+\frac{3}{32} k_{1}+\frac{9}{32} k_{2}\right),
\end{aligned}
$$




$$
\begin{aligned}
& k_{4}=h f\left(x_{i}+\frac{12}{13} h, y_{i}+\frac{1932}{2197} k_{1}-\frac{7200}{2197} k_{2}+\frac{7296}{2197} k_{3}\right), \\
& k_{5}=h f\left(x_{i}+h, y_{i}+\frac{439}{216} k_{1}-8 k_{2}+\frac{3680}{513} k_{3}-\frac{845}{4104} k_{4}\right), \\
& k_{6}=h f\left(x_{i}+\frac{1}{2} h, y_{i}-\frac{8}{27} k_{1}+2 k_{2}-\frac{3544}{2565} k_{3}+\frac{1859}{4104} k_{4}-\frac{11}{40} k_{5}\right),
\end{aligned}
$$

The approximation of the fourth and fifth orders to the previous six steps are:

$$
\begin{aligned}
& y_{i+1}=y_{i}+\frac{25}{216} k_{1}+\frac{1408}{2565} k_{3}+\frac{2197}{4101} k_{4}-\frac{1}{5} k_{5}, \\
& z_{i+1}=y_{i}+\frac{16}{135} k_{1}+\frac{6656}{12825} k_{3}+\frac{28561}{56430} k_{4}-\frac{9}{50} k_{5}+\frac{2}{55} k_{6},
\end{aligned}
$$

with optimal step size, $\delta=0.84\left(\frac{\varepsilon}{R}\right)^{1 / 4}$ where $R=\frac{1}{h}\left|z_{i+1}-y_{i+1}\right|$.

From the flow chart in Figure 2, $x_{0}, y_{0}$ are initial values, $x_{f}$ is final value, $h_{0}$ is initial guess of the step size, $n_{\max }$ is maximum number of iteration and $h_{\min }$ and $h_{\max }$ are minimum and maximum step sizes, respectively.

\section{Validation of the Code}

The tabular and graphical results generated throughout this study are first validated with the formerly published studies to certify the validity of the present numerical code. The numerical values of $-\theta^{\prime}(0) / \sqrt{2}$ are computed for dissimilar Pr values for the limiting cases of $\varepsilon=\lambda=\lambda_{2}=N b=N t=L e=M=N r=B i=f_{w}=0$, as exhibited in Table 1. It is evident that the values generated by Maple code and those tabulated by Bataller [20], Roşca and Pop [23] and Mohamed et al. [24], who implemented the respective RungeKutta method of fourth-order, bvp4c function and Keller-box method are in a good reconciliation, thus confirming the reliability and accuracy of the present codes. 


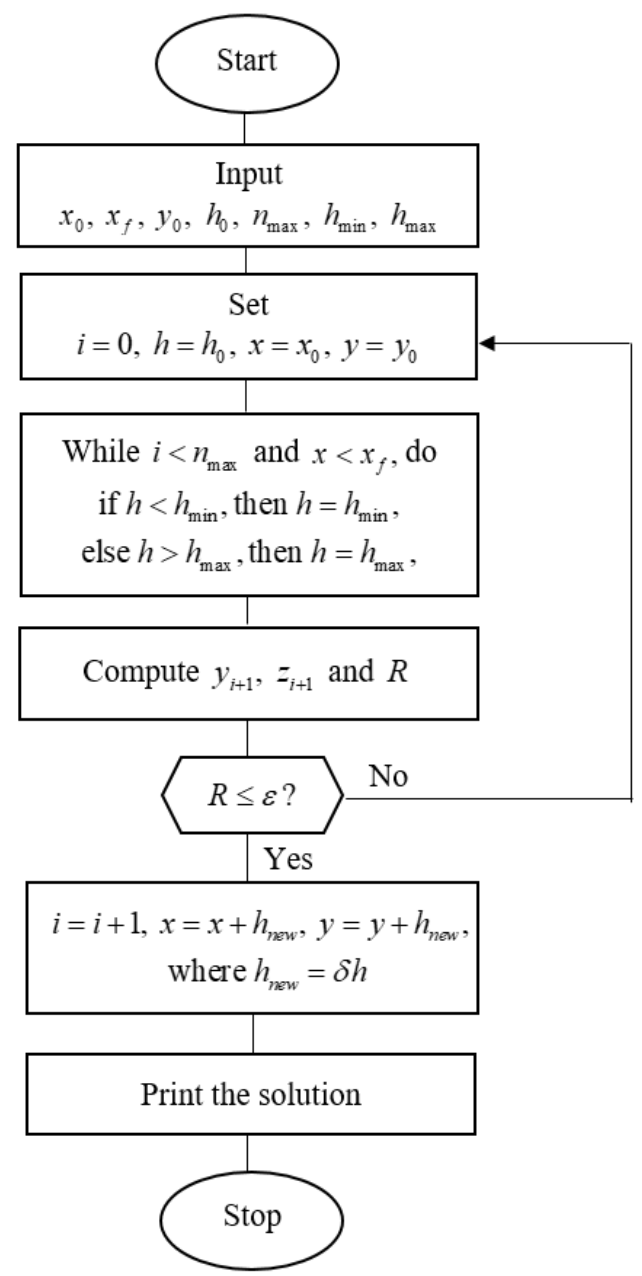

Figure 2. The numerical algorithm for the RKF45 method.

\section{RESULTS AND DISCUSSION}

This section discusses the responses of imperative physical parameters on the specified distributions with the purpose of understanding the insight of the problem. As a whole, the succeeding default parameters are used, unless mentioned otherwise: $\lambda=N b=\varepsilon=0.1, \lambda_{2}=0.2, M=0.7, \operatorname{Pr}=7, N t=L e=B i=0.5, f_{w}=0.3$ and $\mathrm{Nr}=1.0$.

\section{Effect of Parameters on Temperature Profile}

Deborah number, $\lambda_{2}$

Figure 3 (a) discloses the upshot of the Deborah number, $\lambda_{2}$ on the temperature profile. It is perceived that the profile for temperature is a declining function of $\lambda_{2}$. Theoretically, a small Deborah number corresponds to situation where the material is in relaxed state and performs in a viscous manner, while a larger Deborah number corresponds to situation where the material acts relatively elastically. Since $\lambda_{2}$ is directly associated with the relaxation time, increasing $\lambda_{2}$ has reinforced the retardation time. Such reinforcement 
brings about reductions in the temperature accompanied with the thermal boundary layer thickness.

\section{Magnetic parameter, $M$}

Figure 3(b) displays the enhancement of the temperature profile in response to the increasing magnetic parameter, $M$ along the $y$-axis. Here, parameter $M$ implicates the manifestation of electrically conducting fluid that modifies the temperature profile through the polarisation of the fluid. Accordingly, a resistive nature of magnetic force named Lorentz force that has a propensity to retard the fluid flow and captivate nanoparticle to the surface is induced. As $M$ rises, the Lorentz force comes to be stronger, this means enhancing the fluid friction. This enhancement is correspondingly accountable to higher temperature of hydromagnetic flow, $M>0$ compared to the hydrodynamic flow, $M=0$. Hence, increment in the thermal boundary layer flow is foreseeable.

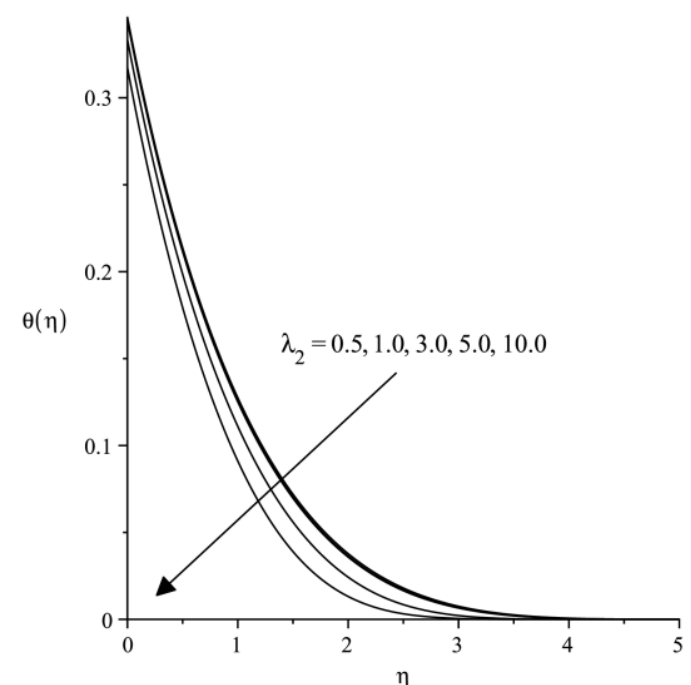

(a)

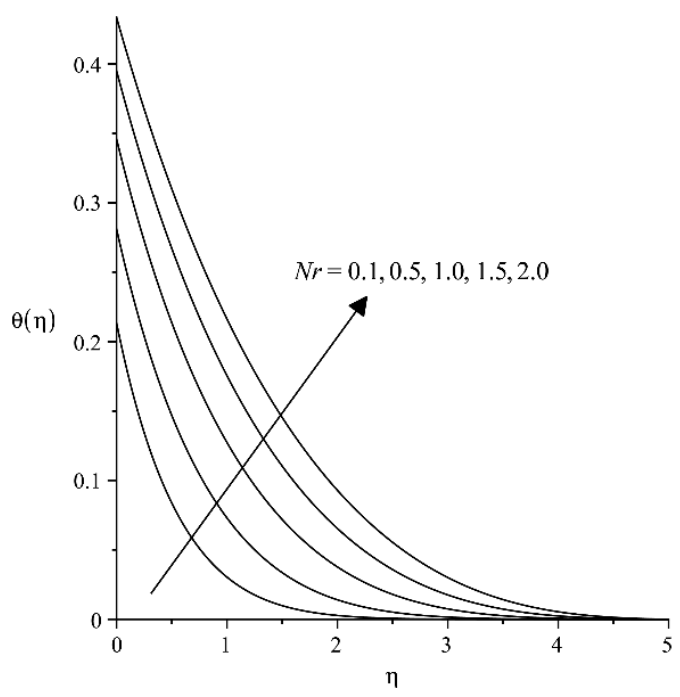

(c)

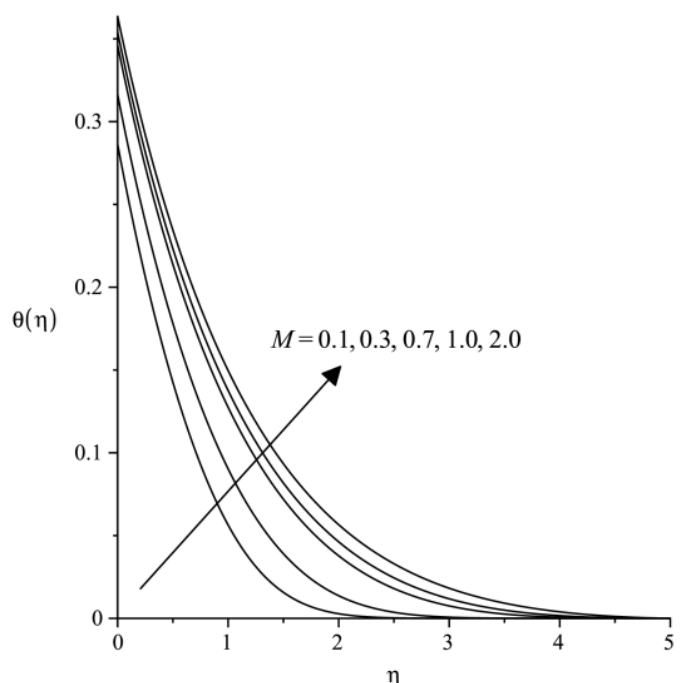

(b)

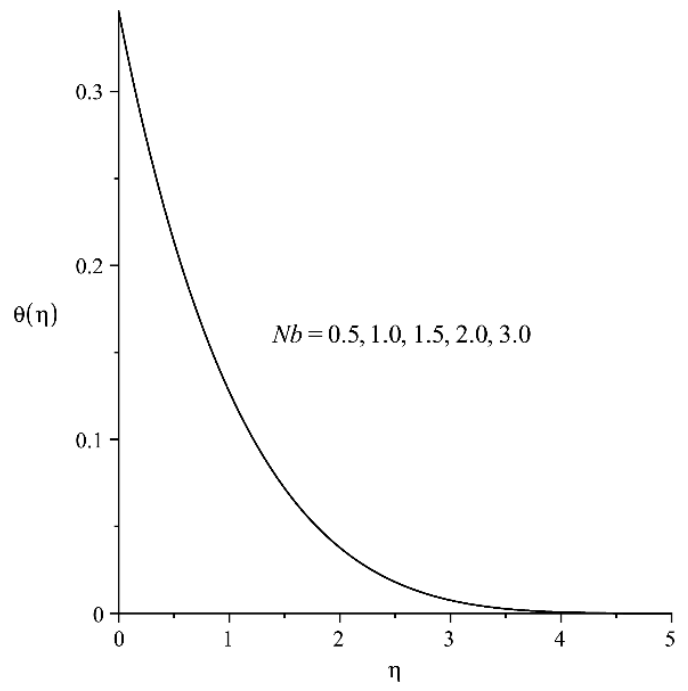

(d) 


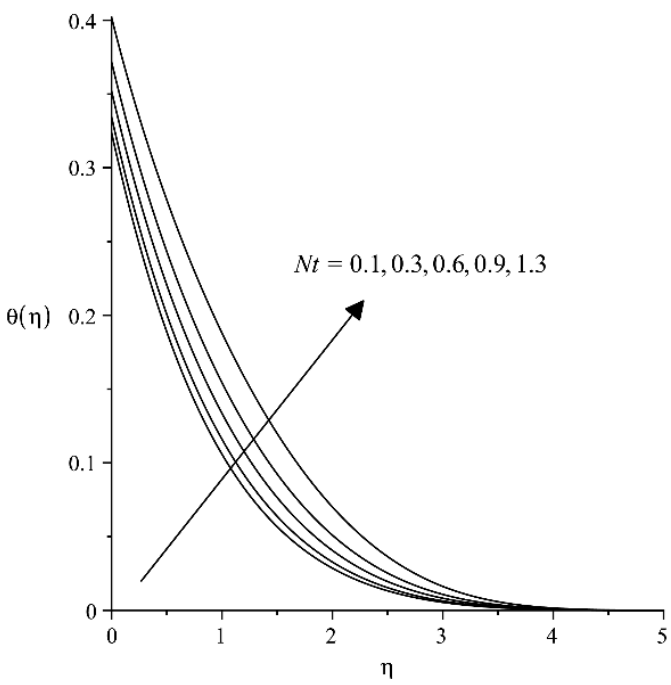

(e)

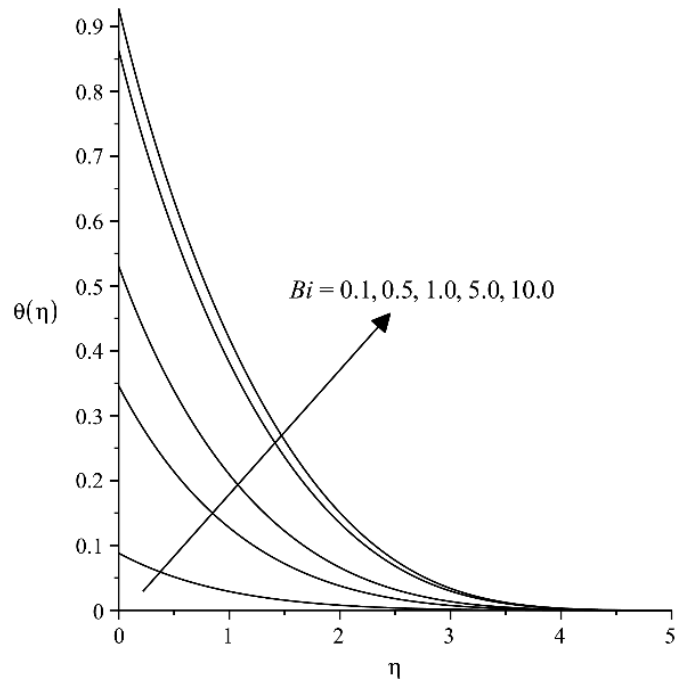

(f)

Figure 3. Temperature profile $\theta(\eta)$ for diverse values of (a) $\lambda_{2}$. (b) $M$ (c) $N r$ (d) $N b$ (e) $\mathrm{Nt}$ and; (f) $\mathrm{Bi}$.

\section{Radiation parameter, $\mathrm{Nr}$}

In Figure 3(c), the temperature profile is inspected over varied values of the radiation parameter, $\mathrm{Nr}$. It is evident from the figure that $\mathrm{Nr}$ exhibits an increasing impact on the temperature profile. This outcome is expected as the presence of $\mathrm{Nr}$ tends to deliver more heat to the surface, thereby thickening the thermal boundary layer. The temperature also varies significantly at the surface, $\eta=0$ by reason of the convectively heated moving plate. Also, a progressive deterioration of the temperature in the direction of the freestream can also be seen when $\eta$ increases.

\section{Brownian motion, $\mathrm{Nb}$}

Figure 3(d) displays the impact of Brownian motion, $N b$ on the temperature profile. Physically, $\mathrm{Nb}$ is defined as random motion of nanoparticles inside the base fluid. A rise in $\mathrm{Nb}$ implies recurring collision between the nanoparticles and this leads to the rapid transfer of inertial forces. By this definition, the temperature profile is likely to enhance owing to the longer engagement of heat in the flow before conveying it to the surrounding [16]. Nevertheless, it is clear from the figure that the increase of $\mathrm{Nb}$ shows negligible changes on the temperature profile. Such circumstance is closely linked to the zero nanoparticle flux condition at the boundary, where temperature turns out to be independent over the Brownian motion [15]. For that reason, it can be understood that the random motion of suspended nanoparticles pronounces insignificant impacts on the temperature.

\section{Thermophoresis diffusion parameter, $\mathrm{Nt}$}

Plot of the temperature profile for the thermophoresis diffusion parameter $N t$ is disclosed in Figure 3(e). The fluid temperature and its related thickness of thermal boundary layer are augmented in consequence of the rising value of Nt. As stated by Zaidi and Mohyud- 
Din [13], higher $N t$ values basically assist in the enhancement of the nanoparticle's momentum on the hotter side. More kinetic energy is produced and conveyed towards a cooler side by reason of larger temperature gradient as well as temperature difference. The transferred kinetic energy warms up the fluid on the cooler side continuously, thereby increasing the temperature.

\section{Biot number, Bi}

Figure 3(f) is sketched to examine the convective heating impact named Biot number, $B i$ on the temperature profile. According to Ibrahim and Haq [14], Bi explains the relationship between the convection at the surface to the conduction inside the surface of a body. As such, improvement of the temperature is related with stronger convection at the surface that promotes deeper penetration of the thermal effect into the fluid. Accordingly, the thermal boundary layer escalates together with the ongoing degeneration of the profile towards the freestream.

\section{Effect of Parameters on Concentration Profile}

\section{Brownian motion, $\mathrm{Nb}$}

Figure 4(a) examines the response of increasing $N b$ values towards the concentration profile. Noticeably, the concentration profile gives negative values when $\eta=0$. This behaviour, which is caused by the curbed nanoparticle flux, has found to be similar as the existing findings disclosed by Kuznetsov and Nield [12], Jahan et al. [17] and Halim et al. [16]. When $0 \leq \eta \leq 0.2$, the concentration profile overshoots and a larger $N b$ value, $N b=3$ is identified to be closer to zero. When $0.2 \leq \eta \leq 5$, the graph reverses and the reduction of nanoparticle concentrations can be explained from the decremented displacement among the nanoparticles following the recurring random collision. This subsequently gives rise to the deprivation of the nanoparticle concentration along with the concentration boundary layer thickness.

\section{Thermophoresis diffusion parameter, $N t$}

Figure 4(b) illustrates a resemblance flipping behaviour of Figure 4(a) but in the opposite manner. It is apparent that the concentration profile is nearly zero in the interval $0 \leq \eta \leq 0.2$ when $N t=0.1$. This is due to the passive control condition where nanoparticle flux is being suppressed and transported to the surrounding to prevent nanoparticle deposition [16]. As for the interval $0.2 \leq \eta \leq 5$, the concentration profile is higher when $N t=1.3$ but lower when $N t=0.1$. Analogous to Figure 3(e), an increase in the temperature difference also prevails upon the dispersion of the nanoparticles towards the cooler environment, hence intensifying the nanoparticle concentration.

\section{Biot number, $B i$}

Figure 4(c) also displays a significant outcome of zero nanoparticle flux condition where the nanoparticle concentration shrinkages near the surface before it flips over to intensify in a similar manner as parameter $N t$ in Figure 4(b). Since temperature is one of the factor 
that drives the nanoparticle concentration, predictably, a larger $B i$ would as well stimulate a deeper penetration of the concentration into the fluid. This prediction is in fact visualized in the specified interval of $0.2 \leq \eta \leq 5$ for the larger value of $B i$.

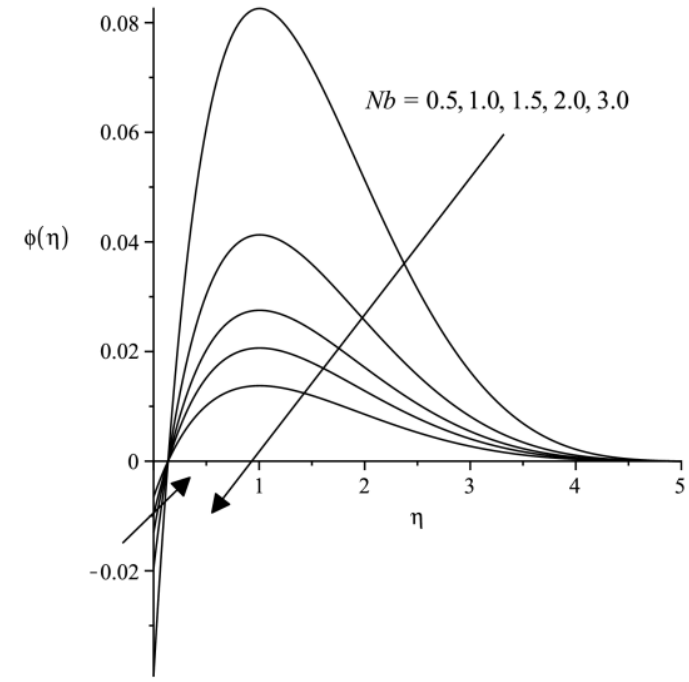

(a)

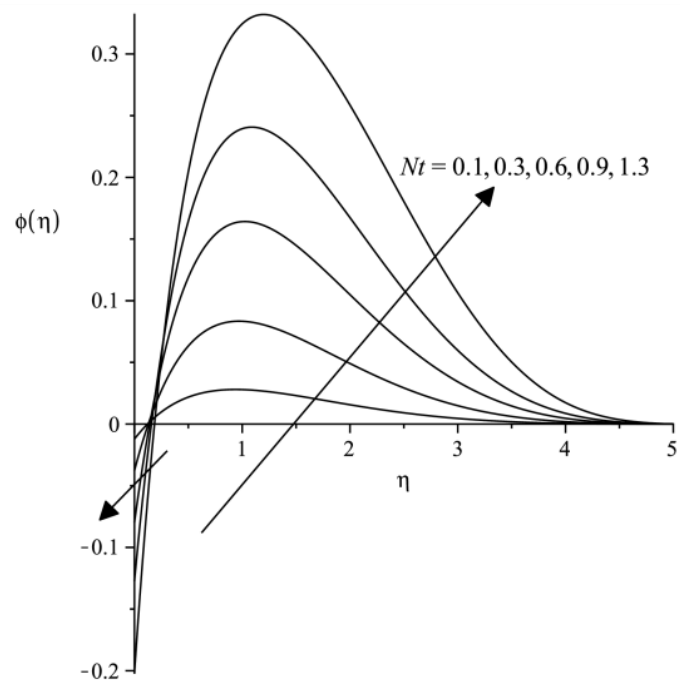

(b)

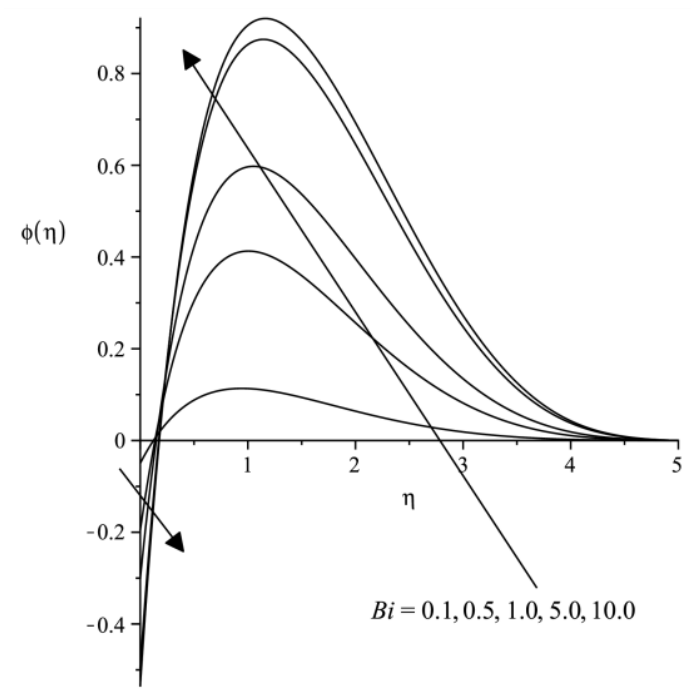

(c)

Figure 4. Nanoparticle concentration $\phi(\eta)$ for diverse values of (a) $N b$, (b) $N t$ and;

(c) $B i$

\section{Effect of Parameters of Engineering Interests}

Variations of the reduced skin friction coefficient $C_{f r}$ and Nusselt number Nur for physical parameters $\lambda_{2}, M, N r, N b, N t$ and $B i$ are provided in Table 2.

Reduced skin friction coefficient, $C_{f r}$ 
From the table, it is obvious that the $C_{f r}$ decelerates with increasing values of $\lambda_{2}$ and $M$. The finding signifies that the fluid friction becomes progressively ineffective following the augmentation of the combined viscous and elastic properties of materials and the magnetic strength. Further, parameters $\mathrm{Nr}, \mathrm{Nb}, \mathrm{Nt}$ and $\mathrm{Bi}$ exhibit no variations on the $C_{f r}$ owing to the decoupled boundary layer Eqs. (23) to (25). These parameters can be simply interpreted as independent of the $C_{f r}$ and can be evidently proven by the existance of a unique solution for the $C_{f r}$, i.e. $f^{\prime \prime}=-0.196880$.

Table 1. Comparative values of $-\theta^{\prime}(0) / \sqrt{2}$ for dissimilar values of $\operatorname{Pr}$ when $B i \rightarrow \infty$

$$
\varepsilon=\lambda=\lambda_{2}=N b=N t=L e=M=N r=f_{w}=0 .
$$

\begin{tabular}{ccccc}
\hline Pr & Bataller [20] & Roșca and Pop [23] & Mohamed et al. [24] & Present \\
\hline 0.7 & 0.29268 & 0.29268 & 0.292680 & 0.292778 \\
0.8 & - & 0.30691 & 0.306917 & 0.307005 \\
1 & - & 0.33205 & 0.332057 & 0.332140 \\
5 & 0.57669 & 0.57668 & 0.576689 & 0.576683 \\
10 & 0.72814 & 0.72814 & 0.728141 & 0.728140 \\
\hline
\end{tabular}

Table 2. Variations of $C_{f r}$ and Nur for diverse values of $\lambda, \lambda_{2}, M, \gamma, N$ and $\alpha_{0}$ when $\lambda=0.1, \operatorname{Pr}=7, L e=0.5, \varepsilon=0.1$ and $f_{w}=0.3$.

\begin{tabular}{cccccccc}
\hline$\lambda_{2}$ & $M$ & $N r$ & $N b$ & $N t$ & $B i$ & $C_{f r}$ & $N u r$ \\
\hline 0.2 & 0.7 & 1.0 & 0.1 & 0.5 & 0.5 & -0.116064 & 0.326909 \\
0.6 & 0.7 & 1.0 & 0.1 & 0.5 & 0.5 & -0.119346 & 0.327570 \\
0.8 & 0.7 & 1.0 & 0.1 & 0.5 & 0.5 & -0.121123 & 0.327837 \\
0.8 & 1.0 & 1.0 & 0.1 & 0.5 & 0.5 & -0.158311 & 0.323621 \\
0.8 & 1.5 & 1.0 & 0.1 & 0.5 & 0.5 & -0.196880 & 0.320499 \\
0.8 & 1.5 & 1.5 & 0.1 & 0.5 & 0.5 & -0.196880 & 0.294131 \\
0.8 & 1.5 & 2.0 & 0.1 & 0.5 & 0.5 & -0.196880 & 0.273836 \\
0.8 & 1.5 & 2.0 & 0.5 & 0.5 & 0.5 & -0.196880 & 0.273836 \\
0.8 & 1.5 & 2.0 & 1.0 & 0.5 & 0.5 & -0.196880 & 0.273836 \\
0.8 & 1.5 & 2.0 & 1.0 & 1.0 & 0.5 & -0.196880 & 0.256585 \\
0.8 & 1.5 & 2.0 & 1.0 & 1.5 & 0.5 & -0.196880 & 0.237603 \\
0.8 & 1.5 & 2.0 & 1.0 & 1.5 & 1.0 & -0.196880 & 0.282986 \\
0.8 & 1.5 & 2.0 & 1.0 & 1.5 & 1.5 & -0.196880 & 0.298536 \\
\hline
\end{tabular}

Reduced Nusselt number, Nur

An increasing impact of parameters $\lambda_{2}$ and $B i$ is detected on the Nur, whereas increasing parameters $M, N r$ and $N t$ act reversely. It is suggested here that the increased values of $\lambda_{2}$ and $B i$ denote a better heat transfer performance for Jeffrey nanofluid when compared with the Newtonian fluid and vice versa for parameters $M, N r$ and $N t$. As for increasing $\mathrm{Nb}$ values, the result is found to be highly relevant with Figure 3(d), where 
the rate of heat transfer continues stagnant by virtue of zero nanoparticle flux condition at the wall and that the nanoparticle concentration there modifies accordingly.

\section{CONCLUSION}

In this work, the flow of Jeffrey nanofluid past a moving plate with the effect of thermal radiation and convective boundary condition is discussed. More physically realistic of passively controlled condition of nanoparticles is incorporated to assume zero nanoparticle flux at the surface. The following conclusions are the main findings of this study:

i. Temperature profile accelerates with the increase of $M, N r, N t$ and $B i$ values while decreases with the increasing $\lambda_{2}$ values.

ii. Nanoparticle concentration is the increasing function of $N t$ and $B i$ and decreasing function of $N b$.

iii. Temperature profile and heat transfer rate are independent of $\mathrm{Nb}$ values due to the zero nanoparticle flux condition at the boundary.

iv. Parameters $N r, N b, N t$ and $B i$ exhibit no variations on the skin friction coefficient due to the decoupled boundary layer Eqs. (23) to (25). Therefore, there exists a unique value of the skin friction coefficient, i.e. $f^{\prime \prime}=-0.196880$.

\section{ACKNOWLEDGEMENT}

The authors are thankful to the Universiti Malaysia Pahang (UMP) for the funding received through PGRS1703100 and RDU170358.

\section{REFERENCE}

[1] Rao AS, Amanulla C, Nagendra N, Bég OA, Kadir A. Hydromagnetic flow and heat transfer in a Williamson Non-Newtonian fluid from a horizontal circular cylinder with Newtonian heating. International Journal of Applied and Computational Mathematics, 2017, 3(4): 3389-3409.

[2] Mohamed MKA, Salleh MZ, Ishak A, Pop I. Stagnation point flow and heat transfer over a stretching/shrinking sheet in a viscoelastic fluid with convective boundary condition and partial slip velocity. The European Physical Journal Plus, 2015, 130(8): 171.

[3] Zokri SM, Arifin NS, Mohamed MKA, Salleh MZ, Kasim ARM, Mohammad NF. Influence of radiation and viscous dissipation on magnetohydrodynamic Jeffrey fluid over a stretching sheet with convective boundary conditions. Malaysian Journal of Fundamental and Applied Sciences, 2017, 13(3): 279-284.

[4] Mohamed MKA, Noar NAZM, Salleh MZ, Ishak A. Free convection boundary layer flow on a horizontal circular cylinder in a nanofluid with viscous dissipation. Sains Malaysiana, 2016, 45(2): 289-296.

[5] Hussanan A, Salleh MZ, Khan I, Tahar RM. Heat and mass transfer in a micropolar fluid with Newtonian heating: an exact analysis. Neural Computing and Applications, 2018, 29(6): 59-67. 
[6] Dalir N, Dehsara M, Nourazar SS. Entropy analysis for magnetohydrodynamic flow and heat transfer of a Jeffrey nanofluid over a stretching sheet. Energy, 2015, 79: 351-362.

[7] Ashraf MB, Hayat T, Alsaedi A, Shehzad SA. Convective heat and mass transfer in MHD mixed convection flow of Jeffrey nanofluid over a radially stretching surface with thermal radiation. Journal of Central South University, 2015, 22(3): 1114-1123.

[8] Narayana PVS, Babu DH. Numerical study of MHD heat and mass transfer of a Jeffrey fluid over a stretching sheet with chemical reaction and thermal radiation. Journal of the Taiwan Institute of Chemical Engineers, 2016, 59(2016): 18-25.

[9] Khan M, Shahid A, Malik MY, Salahuddin T. Thermal and concentration diffusion in Jeffery nanofluid flow over an inclined stretching sheet: A generalized Fourier's and Fick's perspective. Journal of Molecular Liquids, 2018, 251: 7-14.

[10] Zokri SM, Arifin NS, Kasim ARM, Mohammad NF, Salleh MZ. Boundary layer flow over a moving plate in MHD Jeffrey nanofluid: A revised model. In: 2018 Asia-Pacific Conference on Applied Mathematics and Statistics, Hanoi, Vietnam, pp. 02005; 23-26 March, 2018.

[11] Nield D, Kuznetsov A. The Cheng-Minkowycz problem for natural convective boundary-layer flow in a porous medium saturated by a nanofluid. International Journal of Heat and Mass Transfer, 2009, 52(25-26): 5792-5795.

[12] Kuznetsov A, Nield D. The Cheng-Minkowycz problem for natural convective boundary layer flow in a porous medium saturated by a nanofluid: A revised model. International Journal of Heat and Mass Transfer, 2013, 65: 682-685.

[13] Zaidi SZA, Mohyud-Din ST. Convective heat transfer and MHD effects on two dimensional wall jet flow of a nanofluid with passive control model. Aerospace Science and Technology, 2016, 49: 225-230.

[14] Ibrahim W, Haq RU. Magnetohydrodynamic (MHD) stagnation point flow of nanofluid past a stretching sheet with convective boundary condition. Journal of the Brazilian Society of Mechanical Sciences and Engineering, 2016, 38(4): 11551164.

[15] Jahan S, Sakidin H, Nazar R, Pop I. Flow and heat transfer past a permeable nonlinearly stretching/shrinking sheet in a nanofluid: A revised model with stability analysis. Journal of Molecular Liquids, 2017, 233: 211-221.

[16] Halim N, Haq RU, Noor N. Active and passive controls of nanoparticles in Maxwell stagnation point flow over a slipped stretched surface. Meccanica, 2017, 52(7): 1527-1539.

[17] Jahan S, Sakidin H, Nazar R, Pop I. Boundary layer flow of nanofluid over a moving surface in a flowing fluid using revised model with stability analysis. International Journal of Mechanical Sciences, 2017, 131: 1073-1081.

[18] Sakiadis B. Boundary-layer behavior on continuous solid surfaces: I. Boundarylayer equations for two-dimensional and axisymmetric flow. AIChE Journal, 1961, 7(1): 26-28.

[19] Tsou F, Sparrow E, Goldstein RJ. Flow and heat transfer in the boundary layer on a continuous moving surface. International Journal of Heat and Mass Transfer, 1967, 10(2): 219-235.

[20] Bataller RC. Radiation effects in the Blasius flow. Applied Mathematics and Computation, 2008, 198(1): 333-338. 
[21] Bachok N, Ishak A, Pop I. Boundary-layer flow of nanofluids over a moving surface in a flowing fluid. International Journal of Thermal Sciences, 2010, 49(9): $1663-1668$.

[22] Ishak A, Yacob NA, Bachok N. Radiation effects on the thermal boundary layer flow over a moving plate with convective boundary condition. Meccanica, 2011, 46(4): 795-801.

[23] Roşca NC, Pop I. Unsteady boundary layer flow of a nanofluid past a moving surface in an external uniform free stream using Buongiorno's model. Computers \& Fluids, 2014, 95: 49-55.

[24] Mohamed MKA, Noar NAZ, Salleh MZ, Ishak A. Mathematical Model of Boundary Layer Flow over a Moving Plate in a Nanofluid with Viscous Dissipation. Journal of Applied Fluid Mechanics, 2016, 9(5): 2369-2377.

[25] Satish N, Venkatasubbaiah K. Conjugate heat transfer analysis of turbulent forced convection of moving plate in a channel flow. Applied Thermal Engineering, 2016, 100: 987-998.

[26] Soomro FA, Usman M, Ul Haq R, Wang W. Melting heat transfer analysis of Sisko fluid over a moving surface with nonlinear thermal radiation via Collocation method. International Journal of Heat and Mass Transfer, 2018, 126, Part A: 10341042.

[27] Nandeppanavar MM. Melting heat transfer analysis of non-Newtonian Casson fluid due to moving plate. Engineering Computations, 2018, 35(3): 1301-1313.

[28] Afridi MI, Qasim M. Entropy generation and heat transfer in boundary layer flow over a thin needle moving in a parallel stream in the presence of nonlinear Rosseland radiation. International Journal of Thermal Sciences, 2018, 123: 117128.

[29] Narayana PVS, Babu DH. Numerical study of MHD heat and mass transfer of a Jeffrey fluid over a stretching sheet with chemical reaction and thermal radiation. Journal of the Taiwan Institute of Chemical Engineers, 2016, 59: 18-25.

[30] Jaluria Y, Natural convection: Heat and mass transfer. Oxford, England: Pergamon Press; 1980.

[31] Qasim M. Heat and mass transfer in a Jeffrey fluid over a stretching sheet with heat source/sink. Alexandria Engineering Journal, 2013, 52(4): 571-575.

[32] Shercliff JA, Textbook of magnetohydrodynamics. 1st ed. Oxford, England: Pergamon Press; 1965.

[33] Revnic C, Grosan T, Pop I, Ingham DB. Magnetic field effect on the unsteady free convection flow in a square cavity filled with a porous medium with a constant heat generation. International Journal of Heat and Mass Transfer, 2011, 54(9): 1734-1742.

[34] Buongiorno J. Convective transport in nanofluids. Journal of Heat Transfer, 2006, 128(3): 240-250.

[35] Vallado DA, Fundamentals of astrodynamics and applications. 3rd ed. California: Springer Science \& Business Media; 2001.

[36] Press WH, Teukolsky SA, Vetterling WT, Flannery BP, Numerical recipes 3rd edition: The art of scientific computing. 3rd ed. New York: Cambridge University Press; 2007. 